\title{
Other approaches to bivariate ranked set sampling
}

\author{
Mohammad Fraiwan Al-Saleh ${ }^{1, a}$, Hadeel Mohammad Alshboul ${ }^{a}$ \\ ${ }^{a}$ Department of Statistics, Yarmouk University, Jordan
}

\begin{abstract}
Ranked set sampling, as introduced by McIntyre (Australian Journal of Agriculture Research, 3, 385-390, 1952), dealt with the estimation of the mean of one population. To deal with two or more variables, different forms of bivariate and multivariate ranked set sampling were suggested. For a technique to be useful, it should be easy to implement in practice. Bivariate ranked set sampling, as introduced by Al-Saleh and Zheng (Australian \& New Zealand Journal of Statistics, 44, 221-232, 2002), is not easy to implement in practice, because it requires the judgment ranking of each of the combination of the order statistics of the two characteristics. This paper investigates two modifications that make the method easier to use. The first modification is based on ranking one variable and noting the rank of the other variable for one cycle, and do the reverse for another cycle. The second approach is based on ranking of one variable and giving the second variable the same rank (Concomitant Order Statistic) for one cycle and do the reverse for the other cycle. The two procedures are investigated for an estimation of the means of some well-known distributions. It is show that the suggested approaches can be used in practice and can be more efficient than using SRS. A real data set is used to illustrate the procedure.
\end{abstract}

Keywords: ranked set sampling, simple random sampling, bivariate ranked set sampling, bivariate normal distribution, Downton's bivariate exponential distribution, concomitant variable

\section{Introduction}

Statistics is the science of collecting data from a population sample to make an inference about the population. The accuracy of the inference depends on the representativeness of the sample to the population, which is frequently controlled by the size of the sample and the technique used to select it. There are several sampling techniques available for obtaining data with informative image of the large population of interest.

Simple random sampling is the basic method for almost all other sampling techniques. If a sample of size $n$ is drawn from a population of size $N$ (finite population) in such a way that every possible sample of size $n$ has the same chance of being selected, then the sample is called a simple random sample (SRS); the probability of any sample of size $n$ to be the chosen sample is $1 /\left(\begin{array}{l}N \\ n\end{array}\right)$. As a consequence of the definition, all elements of the population have the same inclusion probability, $(=n / N)$. For details about other well-known sampling techniques (Scheaffer et al., 2011).

Ranked set sampling (RSS) was first proposed by McIntyre (1952). This technique of data collection is suitable for situation where taking actual measurements for sample observations is difficult (costly and time-consuming) as compared to the judgment ranking of them. The ranked set sampling technique can be executed as:

\footnotetext{
${ }^{1}$ Corresponding author: Department of Statistics, College of Science, Yarmouk University, Irbid-Jordan.

E-mail: m-saleh@yu.edu.jo

Published 31 May 2018 / journal homepage: http://csam.or.kr

(c) 2018 The Korean Statistical Society, and Korean International Statistical Society. All rights reserved.
} 
1. Randomly draw $m$ SRSs each of size $m$ from the population of interest.

2. The elements within each set are ranked from lowest to largest by judgment with respect to the variable of interest. It is assumed that each element can be ranked visually or by a negligible cost method that does not require actual quantification.

3. From the $t^{\text {th }}$ set, take for actual quantification the element (judgment) ranked as $t^{\text {th }}$ the order statistic.

4. Repeat Steps 1-3 $r$ times (cycles), if necessary, to obtain a RSS of size $n=r m$ units.

The observations of this sample can be denoted by $\left\{X_{(i: m)}^{(j)}: j=1,2, \ldots, r, i=1,2, \ldots, m\right\}$ where $X_{(i: m)}^{(j)}$ is the $t^{\text {th }}$ order statistic from the $t^{\text {th }}$ set of size $m$ in the $j^{\text {th }}$ cycle. When sampling from an infinite population with underlying probability density function (pdf), $f$, and absolutely continuous cumulative distribution function (cdf), $F$, then for each $i, X_{(i:: m)}^{(1)}, X_{(i: m)}^{(2)}, \ldots, X_{(i: m)}^{(r)}$ are independently and identically distributed (iid) $f_{(i: m)}$ with mean $\mu_{(i: m)}$ and variance $\sigma_{(i: m)}^{2}$, while, for each $j, X_{(1: m)}^{(j)}$, $X_{(2: m)}^{(j)}, \ldots, X_{(m: m)}^{(j)}$ are only independent. The pdf of the $t^{t h}$ order statistic is

$$
f_{(i: m)}(x)=\frac{m !}{(i-1) !(m-i) !} F^{i-1}(x)[1-F(x)]^{m-i} f(x) .
$$

In McIntyre's RSS procedure, it is assumed that the researcher could order a set of size units with respect to the characteristic of interest with perfect ranking. He claimed (without proof) that the mean of the ranked set sampling $\hat{\mu}_{\mathrm{RSS}}=(1 /(m r)) \sum_{j=1}^{r} \sum_{i=1}^{m} X_{(i: m)}^{(j)}$, is an unbiased estimator of the population mean $\mu$ regardless of any error in judgment ranking, and in a special unimodal distribution the mean of such a sample is nearly $(m+1) / 2$ times as efficient as the mean of a simple random sample, $\hat{\mu}_{\mathrm{SRS}}=(1 / n) \sum_{j=1}^{m} X_{j}$. The supporting mathematical theory of RSS was provided by Takahasi and Wakimoto (1968). They showed that:

$$
\sum_{i=1}^{m} f_{(i: m))}(x)=f(x), \quad \sum_{i=1}^{m} \mu_{(i: m)}=m \mu, \quad \text { and } \quad m \sigma^{2}=\sum_{i=1}^{m} \sigma_{(i: m)}^{2}+\sum_{i=1}^{m}\left(\mu_{(i: m)}-\mu\right)^{2} .
$$

Based on these identities, $\hat{\mu}_{\text {RSS }}$ is an unbiased estimator of $\mu$ and has smaller variance than $\hat{\mu}_{\text {SRS }}$. They also established the following inequalities for the efficiency of $\hat{\mu}_{\text {RSS }}$ with respect to $\hat{\mu}_{\mathrm{SRS}}$, MSE is mean squared error:

$$
1 \leq \operatorname{eff}\left(\hat{\mu}_{\mathrm{RSS}} ; \hat{\mu}_{\mathrm{SRS}}\right)=\frac{\operatorname{MSE}\left(\hat{\mu}_{\mathrm{SRS}}\right)}{\operatorname{MSE}\left(\hat{\mu}_{\mathrm{RSS}}\right)} \leq \frac{m+1}{2} .
$$

The upper bound is achieved if the underlying distribution is uniform and the lower bound is achieved if it is degenerate.

Al-Saleh and Al-Kadiri (2000) introduced the double ranked set sampling method (DRSS) as a procedure that increases the efficiency of RSS without increasing the set size $m$. Al-Saleh and AlOmari (2002) introduced the multistage ranked set sampling (MSRSS) method as a generalization on DRSS method. The efficiency of estimators using MSRSS for all distributions is found to be between 1 and $m^{2}$.

Moving extreme ranked set sampling (MERSS) is a modification of the usual RSS technique. It was introduced by Al-Odat and Al-Saleh (2001) and investigated further by Al-Saleh and Al-Hadrami (2003a, 2003b). In this procedure, only the extremes values of sets of varied size are identified by judgment. Unlike RSS, MERSS allows for an increase of set size $m$ without introducing too much ranking error. 


\subsection{Bivariate ranked set sampling}

In many real applications such as agriculture or medicine, two variables are closely related and one of them is easy to measure. Stokes (1977) studied RSS with concomitant variables, she assumed that each sampling unit have a bivariate response where $X$ is the character of interest and $Y$ is the concomitant variable that can be easily measured; ranking the variable $X$ can be done according to the variable $Y$. Several authors have considered estimating bivariate characteristics using RSS. McIntyre (1952) suggested applying the RSS procedure to a single selected characteristic and making an assumption regarding the performance of the method for the other characteristics. Patil et al. (1994) investigated two different approaches for dealing with multiple characteristics. In the first approach, as in McIntyre, units were ranked by judgment with respect to one chosen characteristic, and all other characteristics were given the same ranking order as the ranked characteristic. The second approach was based on the concept of size biased permutation. This approach allowed the ranking of units to depend upon several or all characteristics collectively (Norris et al., 1995).

A multivariate version of RSS was introduced by Al-Saleh and Zheng (2002a). For simplicity, the procedure was introduced for two characteristics and referred to it as 'Bivariate ranked set sampling (BVRSS)'. The following steps describe a BVRSS procedure:

1. For a given set size $m$ identify a random sample of size $m^{4}$ from the population and randomly allocate it into $m^{2}$ pools of size $m^{2}$ each, where each pool is a square matrix with $m$ rows and $m$ columns.

2. In the first pool, identify the minimum value by judgment with respect to the first characteristic, for each of the $m$ rows.

3. For the $m$ minima obtained in Step 2, choose the pair that corresponds to the minimum value of the second characteristic, identified by judgment, for actual quantification. This pair, which resembles the label $(1,1)$, is the first element of the BVRSS sample.

4. Repeat Steps 2 and 3 for the second pool, but for actual quantification choose the pair that corresponds to the first minimum value with respect to the first characteristic and the second minimum value with respect to the second characteristic. This pair resembles the label $(1,2)$.

5. Continue the process until the label $(m, m)$ is resembled from the $m^{2}$ (last) pool.

This procedure produces a BVRSS sample of size $m^{2}$.

Ridout (2003) considered several techniques that deals with taking RSS from a population, when we have to deal with multiple characteristics, one method is similar to one of the two methods that we will investigate. His investigation was based on simulation from bivariate normal distribution. Samawi and Al-Saleh (2007) studied relative performance of BVRSS, with respect to RSS and SRS, and investigated the estimation of the population mean using ratio and regression methods. Al-Saleh and Diab (2009) used RSS to estimate the parameters of Downton's bivariate exponential distribution using MERSS with concomitant variable. For more work on RSS see Al-Saleh and Al-Kadiri (2000), Hanandeh and Al-Saleh (2013), Al-Saleh and Zheng (2002b), Al-Saleh and Diab (2009), Al-Saleh and Al-Ananbeh (2007), Al-Saleh and Na' amneh (2014) and Al-Saleh and Aldarabseh (2017), Zamanzade and Mohammadi (2016), Al-Omari and Al-Saleh (2009), Al-Saleh and Ababneh (2015).

BVRSS as introduced by Al-Saleh and Zheng (2002b) can sometimes be difficult to implement in practice. This paper introduces and investigates two modifications of BVRSS. The first modification is the content of Section 2, and the second modification is the content of Section 3. An actual data set is used for illustration. Conclusions and some possible future works are given in Section 4. 
Table 1: A numerical example of the modification with $m=3$

\begin{tabular}{ccccc}
\hline \hline & $(X, Y)$ & $(X, Y)$ & $(X, Y)$ & Steps 1,2,3 \\
\hline \multirow{3}{*}{ Cycle 1 } & $(50,143)$ & $(81,169)$ & $(72,176) \longrightarrow$ & $(50,143)$ \\
& $(77,181)$ & $(67,158)$ & $(64,177) \longrightarrow$ & $(67,158)$ \\
& $(43,147)$ & $(62,155)$ & $(55,163) \longrightarrow$ & $(62,155)$ \\
\hline \multirow{2}{*}{ Cycle 2 } & $(X, Y)$ & $(X, Y)$ & $(X, Y)$ & Steps 4,5,6 \\
& $(52,148)$ & $(83,160)$ & $(71,188) \longrightarrow$ & $(52,148)$ \\
& $(85,189)$ & $(75,169)$ & $(76,174) \longrightarrow$ & $(76,188)$ \\
\hline \hline
\end{tabular}

\section{First modification}

\subsection{First modification of BVRSS (MBVRSS1)}

Suppose $(X, Y)$ is a bivariate random vector with pdf $f(x, y)$. The first suggested approach to obtain a BVRSS can be explained as:

1. Randomly draw a SRS of size from the population of interest; denote the elements of the chosen sample by $\left(X_{1}^{(1)}, Y_{1}^{(1)}\right),\left(X_{2}^{(1)}, Y_{2}^{(1)}\right), \ldots,\left(X_{m}^{(1)}, Y_{m}^{(1)}\right)$. Then, identify the minimum with respect to the $X$-variable and note the rank of the other variable. Denote the identified element by $\left(X_{(1)}^{(1)}, Y_{\left(j_{1}\right)}^{(1)}\right)$ where $j_{1}=1,2, \ldots, m$.

2. Randomly draw another SRS of size, and identify the second minimum with respect to $X$-variable and note the rank of other variable. Denote the identified element by $\left(X_{(2)}^{(2)}, Y_{\left(j_{2}\right)}^{(2)}\right), j_{2}=1,2, \ldots, m$.

3. The process is continued until we identify the maximum with respect to $X$-variable and note the rank of the other variable for a SRS of size $m$. Denote the identified element by $\left(X_{(m)}^{(m)}, Y_{\left(j_{m}\right)}^{(m)}\right)$, $j_{m}=1,2, \ldots, m$.

4. Randomly draw a SRS of size $m$ from the population of interest $\left(X_{1}^{(1) *}, Y_{1}^{(1) *}\right),\left(X_{2}^{(1) *}, Y_{2}^{(1) *}\right), \ldots$, $\left(X_{m}^{(1) *}, Y_{m}^{(1) *}\right)$ then identify the minimum with respect to the $Y$-variable and note the rank of the other variable; denote the identified element by $\left(X_{\left(i_{1}\right)}^{(1) *}, Y_{(1)}^{(1) *}\right)$ where $i_{1}=1,2, \ldots, m$.

5. Randomly draw another SRS of size $m$ and identify the second minimum with respect to the $Y$ variable and note the rank of the other variable; denote the identified element by $\left(X_{\left(i_{2}\right)}^{(2) *}, Y_{(2)}^{(2) *}\right)$ where $i_{2}=1,2, \ldots, m$.

6. The process is continued until we identify the maximum w.r.t. $Y$-variable and note the rank of other variable for a SRS of size $m$, i.e., $\left(X_{\left(m_{2}\right)}^{(m)}, Y_{(m)}^{(m) *}\right), i_{m}=1,2, \ldots, m$.

Steps 1-3 make up the first cycle while steps 4-6 make up the second cycle.

The elements of the set $\left\{\left(X_{\left(i_{k}\right)}^{(k)}, Y_{\left(j_{k}\right)}^{(k)}\right),\left(X_{\left(i_{k}\right)}^{(k) *}, Y_{\left(j_{k}\right)}^{(k) *}\right) ; k=1,2, \ldots, m\right\}$ are the elements of a MBVRSS1 of size $2 \mathrm{~m}$. Cycle 1 and 2 can be repeated $r$ times if needed to obtain a MBVRSS1 of size $n=2 \mathrm{rm}$. Table 1 illustrates the above procedure by a numerical example with $m=3$. The MBVRSS 1 of size 6 is $\{(50,143),(67,158),(62,155),(52,148),(71,188),(76,174)\}$.

\subsection{Estimation of the population mean using MBVRSS1}

Assume that $\left\{\left(X_{\left(i_{k}\right)}^{(k)}, Y_{\left(j_{k}\right)}^{(k)}\right),\left(X_{\left(i_{k}\right)}^{(k) *}, Y_{\left(j_{k}\right)}^{(k) *}\right) ; k=1,2, \ldots, m\right\}$ is a MBVRSS1 of size $2 m$ from $f(x, y)$. Let $\mu_{x}, \mu_{y}, \sigma_{x}, \sigma_{y}$, and $\rho$ be the mean of $X$, the mean of $Y$, the variance of $X$, the variance of $Y$ and the 
correlation between $X$ and $Y$, respectively. We want to use the overall mean to estimate $\mu_{x}$ (for simplicity, we redenote it by $\mu$ ). For a given $\left(i_{1}, i_{2}, \ldots, i_{m}\right)$, let

$$
\hat{\mu}=\frac{\sum_{k=1}^{m} X_{(k)}^{(k)}+\sum_{k=1}^{m} X_{\left(i_{k}\right)}^{(k) *}}{2 m} .
$$

From Takahasi and Wakimoto (1968), we have $\sum_{k=1}^{m} f_{(k)}(x)=m f(x)$. Thus, $E\left(\sum_{k=1}^{m} X_{(k)}^{(k)}\right)=$ $m \int_{-\infty}^{\infty} x f(x) d x=m \mu, E\left(\sum_{k=1}^{m} X_{\left(i_{k}\right)}^{(k) *}\right)=\sum_{k=1}^{m} \mu_{\left(i_{k}\right)}$. Therefore, $E(\hat{\mu})=(\mu / 2)+(1 / 2 m) \sum_{k=1}^{m} \mu_{\left(i_{k}\right)}$. Thus, $\hat{\mu}$ can be a biased estimator. The estimator is unbiased if $i_{k}=k$ for $k=1,2, \ldots, m$. Also,

$$
\operatorname{MSE}(\hat{\mu})=\operatorname{Var}(\hat{\mu})+(\operatorname{Bias}(\hat{\mu}))^{2}=\frac{1}{4 m^{2}}\left[\sum_{k=1}^{m} \sigma_{(k)}^{2}+\sum_{k=1}^{m} \sigma_{\left(i_{k}\right)}^{2}\right]+\left(-\frac{\mu}{2}+\frac{1}{2 m} \sum_{k=1}^{m} \mu_{\left(i_{k}\right)}\right)^{2} .
$$

Also, from Takahasi and Wakimoto (1968), we have $\sum_{k=1}^{m} \sigma_{(k)}^{2}=m \sigma^{2}-\sum_{k}^{m}\left(\mu_{(k)}-\mu\right)^{2}$. Thus,

$$
\operatorname{MSE}(\hat{\mu})=\frac{1}{4 m^{2}}\left[m \sigma^{2}-\sum_{k}^{m}\left(\mu_{(k)}-\mu\right)^{2}+\sum_{k=1}^{m} \sigma_{\left(i_{k}\right)}^{2}\right]+\left(-\frac{\mu}{2}+\frac{1}{2 m} \sum_{k=1}^{m} \mu_{\left(i_{k}\right)}\right)^{2} .
$$

Let $X_{1}, X_{2}, \ldots, X_{2 m}$ is a SRS with common pdf $f(x)$. Let $\hat{\mu}_{\mathrm{SRS}}=\sum_{k=1}^{2 m} X_{k} /(2 m)$, then, then efficiency of $\hat{\mu}$ w.r.t. $\hat{\mu}_{\text {SRS }}$ for a given $\left(i_{1}, i_{2} \ldots, i_{m}\right)$ is

$$
\operatorname{eff}\left(\hat{\mu} ; \hat{\mu}_{\mathrm{SRS}}\right)=\frac{\sigma^{2}}{\frac{1}{2 m}\left[m \sigma^{2}-\sum_{k=1}^{m}\left(\mu_{(k)}-\mu\right)^{2}+\sum_{k=1}^{m} \sigma_{\left(i_{k}\right)}^{2}\right]+2 m\left(-\frac{\mu}{2}+\frac{1}{2 m} \sum_{k=1}^{m} \mu_{\left(i_{k}\right)}\right)^{2}} .
$$

\subsection{Efficiency of the MBVRSS1 for some specific distributions}

In this subsection, we will investigate the performance of this MBVRSS method for estimating the mean of the uniform, exponential \& normal marginal distributions.

- Uniform distribution: Let $X_{1}, X_{2}, \ldots, X_{2 m}$ be a SRS from $U(0, \theta)$, with pdf $f(x)$ and $\operatorname{cdf} F(x)$ :

$$
f(x)=\left\{\begin{array}{ll}
\frac{1}{\theta}, & \text { if } 0<x \leq \theta, \\
0, & \text { otherwise, }
\end{array} \quad F(x)= \begin{cases}0, & x<0 \\
\frac{x}{\theta}, & 0 \leq x \leq \theta \\
1, & x \geq \theta\end{cases}\right.
$$

The pdf of the $k^{\text {th }}$ order statistic, $X_{(k)}$, of a SRS of size $m$ is $f_{(k)}(x)=m ! /\{(k-1) !(m-k) !\}(x / \theta)^{k-1}(1-$ $x / \theta)^{m-k}(1 / \theta)$. It can be shown that $X_{(k)} / \theta$ has the beta distribution with $\alpha=k$ and $\beta=m-k+1$. Thus, $E\left(X_{(k)}\right)=\mu_{(k)}=\{\alpha /(\alpha+\beta)\} \theta=\{k /(m+1)\} \theta$ and $\operatorname{Var}\left(X_{(k)}\right)=\sigma_{(k)}^{2}=\left[\alpha \beta /\left\{(\alpha+\beta)^{2}(\alpha+\beta+1)\right\}\right] \theta^{2}=$ $\left[k(m-k+1) /\left\{(m+1)^{2}(m+2)\right\}\right] \theta^{2}$. For uniform distribution, $U(0, \theta)$, we have $E(X)=\mu=\theta / 2$, $\operatorname{Var}(X)=\sigma^{2}=\theta^{2} / 12$. Thus, $E\left(\hat{\mu}_{\mathrm{SRS}}\right)=\theta / 2$ and $\operatorname{Var}\left(\hat{\mu}_{\mathrm{SRS}}\right)=\theta^{2} /(24 m)$. Therefore, the efficiency of $\hat{\mu}$ w.r.t. $\hat{\mu}_{\mathrm{SRS}}$ for a given $\left(i_{1}, i_{2}, \ldots, i_{m}\right)$ is

$$
\operatorname{eff}\left(\hat{\mu} ; \hat{\mu}_{\mathrm{SRS}}\right)=\frac{\sigma^{2}}{\frac{6}{m}\left[\frac{m}{12}-\sum_{k=1}^{m}\left(\frac{k}{m+1}-\frac{1}{2}\right)^{2}+\sum_{k=1}^{m} \sigma_{\left(i_{k}\right)}^{2}\right]+24 m\left(-\frac{1}{4}+\frac{1}{2 m} \sum_{k=1}^{m} \frac{i_{k}}{m+1}\right)^{2}} .
$$


Table 2: The eff $\left(\hat{\mu} ; \hat{\mu}_{\mathrm{SRS}}\right)$ for uniform distribution

\begin{tabular}{ll|lc|ll}
\hline \hline \multicolumn{1}{c|}{$m=2$} & Efficiency & \multicolumn{1}{c}{$m=3$} & Efficiency & \multicolumn{1}{c}{$m=4$} & Efficiency \\
\hline$i_{1}=i_{2}=1$ & 1.00 & $i_{1}=i_{2}=i_{3}=1$ & 0.63 & $i_{1}=i_{2}=i_{3}=i_{4}=1$ & 0.40 \\
$i_{1}=1, i_{2}=2$ & 1.50 & $i_{1}=1, i_{2}=2, i_{3}=3$ & 2.00 & $i_{1}=1, i_{2}=2, i_{3}=3, i_{4}=4$ & 2.50 \\
$i_{1}=i_{2}=2$ & 1.00 & $i_{1}=3, i_{2}=i_{3}=2$ & 1.54 & $i_{1}=i_{2}=3, i_{3}=4, i_{4}=1$ & 2.17 \\
& & $i_{1}=i_{2}=i_{3}=3$ & 0.63 & $i_{1}=i_{2}=i_{3}=i_{4}=4$ & 0.40 \\
\hline \hline
\end{tabular}

The values of eff $\left(\hat{\mu} ; \hat{\mu}_{\text {SRS }}\right)$ for some values of $m$ and $\left(i_{1}, i_{2}, \ldots, i_{m}\right)$, are given in Table 2 . The best case then occurs when $i_{k}=k$ for $k=1,2, \ldots, m$. In this case, eff $\left(\hat{\mu} ; \hat{\mu}_{\text {SRS }}\right)$ when $m=2,3,4$ are respectively, 1.50, 2.00, and 2.50. These are the same values for the efficiency (eff) of the usual RSS with set size $m$ and sample size $m$. The efficiency is highly depends on the value of $i_{k}$, which highly depends on the relation between the two variables.

- Exponential distribution: Let $X_{1}, X_{2}, \ldots, X_{2 m}$ be a SRS from $\exp (\theta)$, with pdf $f(x)$ and cdf $F(x)$ :

$$
f(x)=\left\{\begin{array}{ll}
\frac{1}{\theta} e^{-\frac{x}{\theta}}, & \text { if } x \geq 0, \\
0, & \text { otherwise, }
\end{array} \quad F(x)= \begin{cases}0, & x<0, \\
1-e^{-\frac{x}{\theta}}, & x \geq 0 .\end{cases}\right.
$$

The pdf of the $k^{\text {th }}$ order statistic of a random sample of size $m$ is:

$$
f_{(k)}(x)=\frac{m !}{(k-1) !(m-k) !}\left(1-e^{-\frac{x}{\theta}}\right)^{k-1}\left(e^{-\frac{x}{\theta}}\right)^{m-k} \frac{1}{\theta} e^{-\frac{x}{\theta}}, \quad x \geq 0 .
$$

If $X$ is $\exp (\theta)$, then $X / \theta$ is $\exp (1) ; E(X)=\mu=\theta$ and $\operatorname{Var}(X)=\sigma^{2}=\theta^{2}$. Thus, $E\left(\hat{\mu}_{\mathrm{SRS}}\right)=\theta / 2$ and $\operatorname{Var}\left(\hat{\mu}_{\mathrm{SRS}}\right)=\theta^{2} / 2 m$.

$$
E\left(X_{(k)}\right)=\mu_{(k)}=\int_{0}^{\infty} x f_{(k)}(x) d x=\int_{0}^{\infty} \frac{x}{\theta} \frac{m !}{(k-1) !(m-k) !}\left(1-e^{-\frac{x}{\theta}}\right)^{k-1}\left(e^{-\frac{x}{\theta}}\right)^{m-k+1} d x .
$$

The above integral can be evaluated for each value of $k$ using Scientific Work Place package. Also,

$$
\operatorname{MSE}(\hat{\mu})=\frac{1}{4 m^{2}}\left(m \theta^{2}-\sum_{k=1}^{m}\left(\mu_{(k)}-\theta\right)^{2}+\sum_{k=1}^{m} \sigma_{\left(i_{k}\right)}^{2}\right)+\left(-\frac{\theta}{2}+\frac{1}{2 m} \sum_{k=1}^{m} \mu_{\left(i_{k}\right)}\right)^{2} .
$$

Thus, the efficiency of $\hat{\mu}$ with respect to $\hat{\mu}_{\text {SRS }}$ for estimating the mean of exponential distribution is

$$
\operatorname{eff}\left(\hat{\mu} ; \hat{\mu}_{\mathrm{SRS}}\right)=\frac{1}{\frac{1}{2 m}\left[m-\sum_{k=1}^{m}\left(\mu_{(k)}-1\right)^{2}+\sum_{k=1}^{m} \sigma_{\left(i_{k}\right)}^{2}\right]+2 m\left(-\frac{1}{2}+\frac{1}{2 m} \sum_{k=1}^{m} \mu_{\left(i_{k}\right)}\right)^{2}} .
$$

For example, if $m=2$, then $\mu_{(1)}=0.5, \mu_{(2)}=1.5, \sigma_{(1)}^{2}=0.25, \sigma_{(2)}^{2}=1.25$. Thus, eff $\left(\hat{\mu} ; \hat{\mu}_{\text {SRS }}\right)=$ $4 / 3$. The values of eff $\left(\hat{\mu} ; \hat{\mu}_{\mathrm{SRS}}\right)$ for some values of $m$ and $\left(i_{1}, i_{2}, \ldots, i_{m}\right)$ are given in Table 3 . The comments on this table are similar to those on Table 2.

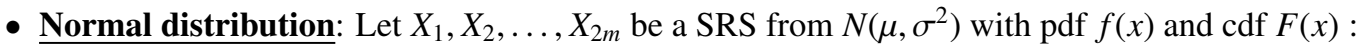

$$
f(x)=\frac{1}{\sigma \sqrt{2 \pi}} e^{\frac{-1}{2 \sigma^{2}}(x-\mu)^{2}}=\frac{1}{\sigma} \phi\left(\frac{x-\mu}{\sigma}\right), \quad F(x)=\Phi\left(\frac{x-\mu}{\sigma}\right), \quad-\infty<x<\infty .
$$


Table 3: The eff( $\left(\hat{\mu} ; \hat{\mu}_{\mathrm{SRS}}\right)$ for exponential distribution

\begin{tabular}{lc|lc|ll}
\hline \hline \multicolumn{1}{c|}{$m=2$} & Efficiency & \multicolumn{1}{c}{$m=3$} & Efficiency & \multicolumn{1}{c}{$m=4$} & Efficiency \\
\hline$i_{1}=i_{2}=1$ & 1.33 & $i_{1}=i_{2}=i_{3}=1$ & 0.97 & $i_{1}=i_{2}=i_{3}=i_{4}=1$ & 0.71 \\
$i_{1}=1, i_{2}=2$ & 1.33 & $i_{1}=1, i_{2}=2, i_{3}=3$ & 1.64 & $i_{1}=1, i_{2}=2, i_{3}=3, i_{4}=4$ & 1.92 \\
$i_{1}=i_{2}=2$ & 0.80 & $i_{1}=3, i_{2}=i_{3}=2$ & 1.44 & $i_{1}=i_{2}=3, i_{3}=4, i_{4}=1$ & 1.71 \\
& & $i_{1}=i_{2}=i_{3}=3$ & 0.49 & $i_{1}=i_{2}=i_{3}=i_{4}=4$ & 0.30 \\
\hline \hline
\end{tabular}

Table 4: The eff $\left(\hat{\mu} ; \hat{\mu}_{\text {SRS }}\right)$ for normal distribution

\begin{tabular}{ll|lc|lc}
\hline \hline \multicolumn{1}{c|}{$m=2$} & Efficiency & \multicolumn{1}{c}{$m=3$} & Efficiency & \multicolumn{1}{c}{$m=4$} & Efficiency \\
\hline$i_{1}=i_{2}=1$ & 1.00 & $i_{1}=i_{2}=i_{3}=1$ & 0.62 & $i_{1}=i_{2}=i_{3}=i_{4}=1$ & 0.30 \\
$i_{1}=1, i_{2}=2$ & 1.47 & $i_{1}=1, i_{2}=2, i_{3}=3$ & 1.91 & $i_{1}=1, i_{2}=2, i_{3}=3, i_{4}=4$ & 2.35 \\
$i_{1}=i_{2}=2$ & 1.00 & $i_{1}=3, i_{2}=i_{3}=2$ & 1.60 & $i_{1}=i_{2}=3, i_{3}=4, i_{4}=1$ & 1.94 \\
& & $i_{1}=i_{2}=i_{3}=3$ & 0.62 & $i_{1}=i_{2}=i_{3}=i_{4}=4$ & 0.39 \\
\hline \hline
\end{tabular}

The pdf of the $k^{\text {th }}$ order statistic of a random sample of size $m$ is

$$
f_{(k)}(x)=\frac{m !}{(k-1) !(m-k) !}\left(\Phi\left(\frac{x-\mu}{\sigma}\right)\right)^{k-1}\left(1-\Phi\left(\frac{x-\mu}{\sigma}\right)\right)^{m-k} \frac{1}{\sigma} \phi\left(\frac{x-\mu}{\sigma}\right) .
$$

Thus,

$$
\operatorname{MSE}(\hat{\mu})=\frac{1}{4 m^{2}}\left(m \sigma^{2}-\sum_{k=1}^{m}\left(\mu_{(k)}-\mu\right)^{2}+\sum_{k=1}^{m} \sigma_{\left(i_{k}\right)}^{2}\right)+\left(-\frac{\mu}{2}+\frac{1}{2 m} \sum_{k=1}^{m} \mu_{\left(i_{k}\right)}\right)^{2}
$$

The value of efficiency of normal distribution does not depend on the parameters $\mu$ and $\sigma^{2}$, so we can find $\mu_{(k)}$ based on standard normal distribution. Thus,

$$
\operatorname{eff}\left(\hat{\mu} ; \hat{\mu}_{\mathrm{SRS}}\right)=\frac{1}{\frac{1}{2 m}\left[m-\sum_{k=1}^{m} \mu_{(k)}^{2}+\sum_{k=1}^{m} \sigma_{\left(i_{k}\right)}^{2}\right]+2 m\left(\frac{1}{2 m} \sum_{k=1}^{m} \mu_{\left(i_{k}\right)}\right)^{2}}
$$

The values of eff $\left(\hat{\mu} ; \hat{\mu}_{\mathrm{SRS}}\right)$ for some values of $m$ and $\left(i_{1}, i_{2}, \ldots, i_{m}\right)$ are given in Table 4 . Again, the best scenario occurs when $i_{k}=k$ for $k=1,2, \ldots, m$.

\subsection{Overall performance of the proposed BVRSS}

In the previous subsection, the efficiency was investigated for some specific values of $\left(i_{1}, i_{2}, \ldots, i_{m}\right)$. In general, $i_{1}, i_{2}, \ldots, i_{m}$ are independent random variables, with $i_{k}=1,2, \ldots, m$. The best case as seen is $i_{k}=k, k=1,2, \ldots, m$, which occurs when the two variables of interest are highly correlated. In this extreme case, we have two RSS each of size $m$. So, in this case if we use $X_{(k)}^{(k)}, X_{\left(i_{k}\right)}^{(k) *}$ to estimate $\mu$, then

$$
\begin{aligned}
\hat{\mu} & =\frac{\sum_{k=1}^{m} X_{(k)}^{(k)}+\sum_{k=1}^{m} X_{\left(i_{k}\right)}^{(k) *}}{2 m} \\
E(\hat{\mu}) & =\frac{1}{2 m}\left(\sum_{k=1}^{m} E\left(X_{(k)}^{(k)}\right)+\sum_{k=1}^{m} E\left(X_{\left(i_{k}\right)}^{(k) *}\right)\right)=\frac{1}{2 m}(m \mu+m \mu)=\mu ;
\end{aligned}
$$


Table 5: The efficiency in general with the best and worst cases eff $\left(\hat{\mu} ; \hat{\mu}_{\mathrm{SRS}}\right)$

\begin{tabular}{ccccccc}
\hline \hline$m$ & 2 & 3 & 4 & 5 & 6 & General $m$ \\
\hline Best case & 1.5 & 2.00 & 2.50 & 3.0 & 3.50 & $(m+1) / 2$ \\
Worst case & 1.2 & 1.33 & 1.43 & 1.5 & 1.56 & $(2 m+2) /(m+3)$ \\
\hline \hline
\end{tabular}

thus, $\hat{\mu}$ is an unbiased estimator of $\mu$. Also,

$$
\operatorname{Var}(\hat{\mu})=\frac{1}{4}\left(\operatorname{Var}\left(\hat{\mu}_{\mathrm{RSS}}\right)+\operatorname{Var}\left(\hat{\mu}_{\mathrm{RSS}}^{*}\right)\right)=\frac{1}{2} \operatorname{Var}\left(\hat{\mu}_{\mathrm{RSS}}\right) .
$$

$\hat{\mu}_{\mathrm{RSS}}$ is the estimator of based on RSS of size $m$. Thus, the efficiency of $\hat{\mu}$ w.r.t. $\hat{\mu}_{\text {SRS }}$ for estimating the mean is

$$
\operatorname{eff}\left(\hat{\mu} ; \hat{\mu}_{\mathrm{SRS}}\right)=\frac{\sigma^{2} / 2 m}{0.5 \operatorname{Var}\left(\hat{\mu}_{\mathrm{RSS}}\right)}=\frac{\sigma^{2} / m}{\operatorname{Var}\left(\hat{\mu}_{\mathrm{RSS}}\right)}=\mathrm{eff},
$$

which is the efficiency of RSS of size $m$ w.r.t. SRS of the same size. It is well known in this case that $1 \leq \operatorname{eff}\left(\hat{\mu} ; \hat{\mu}_{\text {SRS }}\right) \leq(1 / 2)(m+1)$; this means the number of cycles has no effect on the efficiency. The worst case occurs when there is no relation between the two variables; for this case $P\left(i_{1}=1\right)=$ $P\left(i_{2}=2\right)=\cdots=P\left(i_{k}=k\right)=1 / m$, for $k=1,2, \ldots, m$. The resulting sample consists of a RSS and a SRS of size $m$ each, i.e., $X_{\left(i_{k}\right)}^{(k) *}, k=1, \ldots, m$, are equivalent to a SRS of size $m$ :

$$
\begin{aligned}
\operatorname{Var}(\hat{\mu}) & =\frac{1}{4}\left(\operatorname{Var}\left(\hat{\mu}_{\mathrm{RSS}}\right)+\operatorname{Var}\left(\hat{\mu}_{\mathrm{SRS}}^{*}\right)\right) \\
\operatorname{eff}\left(\hat{\mu} ; \hat{\mu}_{\mathrm{SRS}}\right) & =\frac{\sigma^{2} / 2 m}{\frac{1}{4}\left(\operatorname{Var}\left(\hat{\mu}_{\mathrm{RSS}}\right)+\operatorname{Var}\left(\hat{\mu}_{\mathrm{SRS}}^{*}\right)\right)}=\frac{2 \sigma^{2} / m}{\operatorname{Var}\left(\hat{\mu}_{\mathrm{RSS}}\right)} /\left(1+\frac{\operatorname{Var}\left(\hat{\mu}_{\mathrm{SRS}}\right)}{\operatorname{Var}\left(\hat{\mu}_{\mathrm{RSS}}\right)}\right)=\frac{2 \mathrm{eff}}{1+\mathrm{eff}},
\end{aligned}
$$

where eff is the efficiency of the usual RSS of set size $m$ w.r.t. a SRS of size $m$. Since, $1 \leq$ eff $\leq$ $(1 / 2)(m+1)$, we have, $1 \leq \operatorname{eff}\left(\hat{\mu} ; \hat{\mu}_{\text {SRS }}\right) \leq(2 m+2) /(m+3)$. Table 5 contains the values of the efficiency in general for the best and worst cases.

\section{Second modification}

\subsection{Second modification of BVRSS (MBVRSS2)}

In this section, we consider another modification of BVRSS, MBVRSS2. In this modification, we order with respect to the first variable and give the second variable the same rank (concomitant variable) for one cycle and do the other way around for the other cycle. Let $(X, Y)$ be a bivariate random vector with joint pdf, $f(x, y)$. The suggested approach of BVRSS is obtained based on concomitant variable using the following steps:

1. Randomly draw a SRS of size $m$ from the population of interest. Then, identify the minimum with respect to $X$-variable and give the corresponding $Y$-variable the same rank. Denote the identified element by $\left(X_{(1)}^{(1)}, Y_{[1]}^{(1)}\right)$.

2. Randomly draw another SRS of size $m$. Then, identify the second minimum with respect to $X$ variable and give the corresponding $Y$-variable the same rank. Denote the identified element by $\left(X_{(2)}^{(2)}, Y_{[2]}^{(2)}\right)$. 
3. The process is continued until we identify the maximum with respect to $X$-variable and give the corresponding $Y$-variable the same rank for a SRS of size $m$. Denote the identified element by $\left(X_{(m)}^{(m)}, Y_{[m]}^{(m)}\right)$.

4. Randomly draw a SRS of size $m$ from the population of interest. Then, identify the minimum with respect to $Y$-variable and give the corresponding $X$-variable the same rank. Denote the identified element by $\left(X_{[1]}^{(1) *}, Y_{(1)}^{(1) *}\right)$.

5. Randomly draw a SRS of size $m$ from the population of interest. Then, identify the second minimum with respect to $Y$-variable and give the corresponding $X$-variable the same rank. Denote the identified element by $\left(X_{[2]}^{(2) *}, Y_{(2)}^{(2) *}\right)$.

6. The process is continued until we identify the maximum with respect to $Y$-variable and give the corresponding $X$-variable the same rank for a SRS of size $m$. Denote the identified element by $\left(X_{[m]}^{(m) *}, Y_{(m)}^{(m) *}\right)$.

\section{* Steps 1-3 make up the first cycle while steps 4-6 make up the second cycle.}

The elements of the set $\left\{\left(X_{(k)}^{(k)}, Y_{[k]}^{(k)}\right),\left(X_{[k]}^{(k) *}, Y_{(k)}^{(k) *}\right): k=1,2, \ldots, m\right\}$ are the elements of the MBVRSS2 of size $2 m$ using concomitant variable. Cycles 1 and 2 can be repeated $r$ times if needed to obtain a MBVRSS2 of size $n=2 \mathrm{rm}$. The round bracket on a subscript is used to indicate that the ordering is perfect, while the square bracket is used to indicate that the ordering is imperfect.

Consider $\left(X_{[k]}^{(k) *}, Y_{(k)}^{(k) *}\right), k=1,2, \ldots, m$. Yang (1977) has shown that the conditional distribution of the concomitant variable $X_{[k]}^{(k) *}$ given the order statistic $Y_{(k)}^{(k) *}$ is the same as the conditional distribution of $X$ given $Y$; i.e.,

$$
f_{X_{[k]}^{(k) *} \mid Y_{(k)}^{(k) *}}\left(x_{[k]}^{(k) *} \mid y_{(k)}^{(k) *}\right)=f_{X \mid Y}\left(x_{[k]}^{(k) *} \mid y_{(k)}^{(k) *}\right) .
$$

Based on this result we can obtain the mean and the variance of the concomitant order statistic $X_{[k]}^{(k) *}$, in terms of the mean and variance of the order statistic $Y_{(k)}^{(k) *}$.

The performance of this MBVRSS2 is investigated for two important distributions: the Bivariate Normal Distribution and Downton's Bivariate Exponential Distribution.

- Bivariate normal distribution: Assume that a random vector $(X, Y)$ has a bivariate normal distribution which is denoted by $\mathrm{BN}\left(\mu_{x}, \mu_{y}, \sigma_{x}^{2}, \sigma_{y}^{2}, \rho\right)$, where $-\infty<x, y, \mu_{x}, \mu_{y}<\infty, 0<\sigma_{x}^{2}, \sigma_{y}^{2}<\infty$, and $-1<\rho<1 . \mu_{x}, \mu_{y}, \sigma_{x}^{2}, \sigma_{y}^{2}, \rho$ are respectively the mean of $X$, the mean of $Y$, the variance of $X$, the variance of $Y$ and the correlation between $X$ and $Y$. The joint pdf is given by:

$$
f_{X, Y}(x, y)=\frac{1}{2 \pi \sigma_{x} \sigma_{y}} e^{\frac{-1}{2\left(1-\rho^{2}\right)}\left(\left(\frac{x-\mu_{x}}{\sigma_{x}}\right)^{2}-2 \rho\left(\frac{x-\mu_{x}}{\sigma_{x}}\right)\left(\frac{y-\mu_{y}}{\sigma_{y}}\right)+\left(\frac{y-\mu_{y}}{\sigma_{y}}\right)^{2}\right)} .
$$

It is well- known that the marginals are normal and the conditionals are also normal. In particular:

$$
Y=N\left(\mu_{y}, \sigma_{y}^{2}\right) \quad \text { and } \quad(X \mid Y=y)=N\left(\mu_{x}+\rho \frac{\sigma_{x}}{\sigma_{y}}\left(y-\mu_{y}\right), \sigma_{x}^{2}(1-\rho)^{2}\right) .
$$

Using the results of Yang (1977), we have

$$
f_{X_{[k]}^{(k) *} \mid Y_{(k)}^{(k) *}}\left(x_{[k]}^{(k) *} \mid y_{(k)}^{(k) *}\right)=f_{X \mid Y}\left(x_{[k]}^{(k) *} \mid y_{(k)}^{(k) *}\right) .
$$


Table 6: The eff $\left(\hat{\mu}_{x} ; \hat{\mu}_{\mathrm{SRS}}\right)$ for Bivariate normal distribution

\begin{tabular}{ccccccc}
\hline \hline$m \downarrow|\rho| \rightarrow$ & 0.0 & 0.2 & 0.4 & 0.6 & 0.8 & $|\rho| \rightarrow 1$ \\
\hline 2 & 1.19 & 1.20 & 1.23 & 1.28 & 1.36 & 1.47 \\
3 & 1.31 & 1.33 & 1.38 & 1.48 & 1.64 & 1.92 \\
4 & 1.40 & 1.46 & 1.50 & 1.64 & 1.90 & 2.35 \\
\hline \hline
\end{tabular}

Thus,

$$
E\left(X_{[k]}^{(k) *} \mid y_{(k)}^{(k) *}\right)=\mu_{x}+\rho \frac{\sigma_{x}}{\sigma_{y}}\left(y_{(k)}^{(k) *}-\mu_{y}\right) \quad \text { and } \quad \operatorname{Var}\left(X_{[k]}^{(k) *} \mid y_{(k)}^{(k) *}\right)=\sigma_{x}^{2}(1-\rho)^{2}
$$

Therefore,

$$
E\left(X_{[k]}^{(k) *}\right)=E\left(E\left(X_{[k]}^{(k) *} \mid Y_{(k)}^{(k) *}\right)\right)=\mu_{x}+\rho \frac{\sigma_{x}}{\sigma_{y}} E\left(Y_{(k)}^{(k) *}-\mu_{y}\right)
$$

also,

$$
\operatorname{Var}\left(X_{[k]}^{(k) *}\right)=\operatorname{Var}\left(E\left(X_{[k]}^{(k) *} \mid Y_{(k)}^{(k) *}\right)\right)+E\left(\operatorname{Var}\left(X_{[k]}^{(k) *} \mid Y_{(k)}^{(k) *}\right)\right)=\sigma_{x}^{2}(1-\rho)^{2}+\rho^{2} \frac{\sigma_{x}^{2}}{\sigma_{y}^{2}} \operatorname{Var}\left(Y_{(k)}^{(k) *}\right) .
$$

Let

$$
\hat{\mu}_{x}=\frac{\sum_{k=1}^{m} X_{(k)}^{(k)}+\sum_{k=1}^{m} X_{\left(i_{k}\right)}^{(k) *}}{2 m}
$$

then it can be shown easily that

$E\left(\hat{\mu}_{x}\right)=\mu_{x} \quad$ and $\quad \operatorname{Var}\left(\hat{\mu}_{x}\right)=\frac{1}{4 m^{2}}\left(m \sigma_{x}^{2}-\sum_{k=1}^{m}\left(\mu_{(k)}-\mu\right)^{2}+m \sigma_{x}^{2}\left(1-\rho^{2}\right)+\rho^{2} \frac{\sigma_{x}^{2}}{\sigma_{y}^{2}} \sum_{k=1}^{m} \operatorname{Var}\left(Y_{(k)}^{(k) *}\right)\right)$.

Let $X_{1}, X_{2}, \ldots, X_{2 m}$ be a SRS from $N\left(\mu_{x}, \sigma_{x}^{2}\right)$ and let $\hat{\mu}_{\mathrm{SRS}}=\bar{X}$. Then

$$
\begin{aligned}
\operatorname{eff}\left(\hat{\mu}_{x} ; \hat{\mu}_{\mathrm{SRS}}\right) & =\frac{\sigma_{x}^{2} / 2 m}{\frac{1}{4 m^{2}}\left(m \sigma_{x}^{2}-\sum_{k=1}^{m}\left(\mu_{(k)}-\mu\right)^{2}+m \sigma_{x}^{2}\left(1-\rho^{2}\right)+\rho^{2} \frac{\sigma_{x}^{2}}{\sigma_{y}^{2}} \sum_{k=1}^{m} \operatorname{Var}\left(Y_{(k)}^{(k) *}\right)\right)} \\
& =\frac{2 m}{(m / \mathrm{eff})+m\left(1-\rho^{2}\right)+\rho^{2}(m / \mathrm{eff})} \\
& =\frac{2 \mathrm{eff}}{1+\operatorname{eff}\left(1-\rho^{2}\right)+\rho^{2}} .
\end{aligned}
$$

Table 6 gives the efficiency for $m=2,3,4$ and different values of $\rho$. The efficiency is always larger than 1 ; it is increasing in $m$ for fixed $\rho$, and increasing in $\rho$ for fixed $m$. As $|\rho| \rightarrow 1$, the efficiency goes to eff, which is the efficiency of $\hat{\mu}_{\text {RSS }}$ with respect to $\hat{\mu}_{\text {SRS }}$ for estimating the mean of normal distribution. 
Table 7: The eff $\left(\hat{\mu}_{x} ; \hat{\mu}_{\mathrm{SRS}}\right)$ for Downton's bivariate exponential distribution

\begin{tabular}{ccccccc}
\hline \hline$m \downarrow|\rho| \rightarrow$ & 0 & 0.2 & 0.4 & 0.6 & 0.8 & $|\rho| \rightarrow 1$ \\
\hline 2 & 1.14 & 1.15 & 1.17 & 1.20 & 1.26 & 1.33 \\
3 & 1.25 & 1.30 & 1.36 & 2.04 & 1.47 & 1.64 \\
4 & 1.32 & 1.33 & 1.38 & 1.48 & 1.64 & 1.92 \\
\hline \hline
\end{tabular}

- Downton's bivariate exponential distribution: This distribution, denoted by $\operatorname{DBE}\left(\theta_{x}, \theta_{y}, \rho\right)$, was introduced by Downton (1970). Its pdf is given by:

$$
f_{X, Y}(x, y)=\frac{1}{\theta_{x} \theta_{y} \rho} e^{-\left(\frac{x}{\theta_{x}(1-\rho)}+\frac{y}{\theta_{y}(1-\rho)}\right) \times I_{0}\left(\frac{2(\sqrt{\rho x y})}{(1-\rho) \sqrt{\theta_{x} \theta_{y}}}\right)}
$$

where, $x, y, \theta_{x}, \theta_{y}>0,0 \leq \rho<1$, and $I_{0}(z)=\sum_{k=1}^{\infty}(z / 2)^{2 k} / k !^{2}$ is the modified Bessel function of the first kind of order zero.

The marginal distributions of $X$ and $Y$ are exponential with parameters $\theta_{x}$ and $\theta_{y}$, respectively. Also,

$$
E(X \mid Y=y)=(1-\rho) \theta_{x}+\rho \frac{\theta_{x}}{\theta_{y}} y \quad \text { and } \quad \operatorname{Var}(X \mid Y=y)=(1-\rho)^{2} \theta_{x}^{2}+2 \rho(1-\rho) \frac{\theta_{x}^{2}}{\theta_{y}^{2}} y .
$$

Note that unlike the bivariate normal distribution, the conditional variance depends on the given value of $Y$. Using the results of Yang (1977), we have

$$
f_{X_{[k]}^{(k) *} \mid Y_{(k)}^{(k) *}}\left(x_{[k]}^{(k) *} \mid y_{(k)}^{(k) *}\right)=f_{X \mid Y}\left(x_{[k]}^{(k) *} \mid y_{(k)}^{(k) *}\right) .
$$

Thus,

$$
E\left(X_{[k]}^{(k) *} \mid y_{(k)}^{(k) *}\right)=(1-\rho) \theta_{x}+\rho \frac{\theta_{x}}{\theta_{y}} Y_{(k)}^{(k) *} \quad \text { and } \quad \operatorname{Var}\left(X_{[k]}^{(k) *} \mid y_{(k)}^{(k) *}\right)=(1-\rho)^{2} \theta_{x}^{2}+2 \rho(1-\rho) \frac{\theta_{x}^{2}}{\theta_{y}^{2}} Y_{(k)}^{(k) *}
$$

therefore

$$
E\left(X_{[k]}^{(k) *}\right)=E\left(E\left(X_{[k]}^{(k) *} \mid Y_{(k)}^{(k) *}\right)\right)=(1-\rho) \theta_{x}+\rho \frac{\theta_{x}}{\theta_{y}} E\left(Y_{(k)}^{(k) *}\right)
$$

also,

$$
\operatorname{Var}\left(X_{[k]}^{(k) *}\right)=\operatorname{Var}\left(E\left(X_{[k]}^{(k) *} \mid Y_{(k)}^{(k) *}\right)\right)+E\left(\operatorname{Var}\left(X_{[k]}^{(k) *} \mid Y_{(k)}^{(k) *}\right)\right)=(1-\rho)^{2} \theta_{x}^{2}+\rho^{2} \frac{\theta_{x}^{2}}{\theta_{y}^{2}} \operatorname{Var}\left(Y_{(k)}^{(k) *}\right) .
$$

Thus, $\hat{\theta}_{x}=\left\{\sum_{k=1}^{m} X_{(k)}^{(k)}+\sum_{k=1}^{m} X_{\left(i_{k}\right)}^{(k) *}\right\} / 2 m$, as in the bivariate normal case, is an unbiased estimator for $\theta_{x}$. The efficiency of $\hat{\theta}_{x}$ w.r.t. $\hat{\theta}_{\text {SRS }}$ is

$$
\operatorname{eff}\left(\hat{\theta}_{x} ; \hat{\theta}_{\mathrm{SRS}}\right)=\frac{2 \mathrm{eff}}{1+\operatorname{eff}\left(1-\rho^{2}\right)+\rho^{2}} .
$$

From Table 7, the efficiency is always larger than 1, increasing in $m$ for fixed $\rho$, and increasing in $\rho$ for fixed $m$. Also as $|\rho| \rightarrow 1$, the efficiency goes to eff, which is the efficiency of $\hat{\mu}_{\text {RSs }}$ with respect to $\hat{\mu}_{\text {SRS }}$ for estimating the mean of the exponential distribution. 


\subsection{General setting of the technique}

In the previous subsection, we investigated the efficiency of $\hat{\mu}_{x}$ with respect to $\hat{\mu}_{\mathrm{SRS}}$ for the mean of some well-known distributions. In this subsection, we will look at a general case.

Let $\left\{\left(X_{(k)}^{(k)}, Y_{[k]}^{(k)}\right),\left(X_{[k]}^{(k) *}, Y_{(k)}^{(k) *}\right): k=1,2, \ldots, m\right\}$ be a MBVRSS2 of size $2 m$ obtained using the suggested technique explained above. Frey (2007) proposed that the distribution of the concomitant order statistic can be written as a linear combination of the distribution of the order statistics:

$$
X_{[k]}^{(k) *} \stackrel{d}{=} \sum_{i=1}^{m} a_{i}^{(k)} X_{(i)},
$$

where for each value of $k, a_{i}^{(k)} \geq 0$, and $\sum_{i=1}^{m} a_{i}^{(k)}=1$ (So $X_{[k]}^{(k) *}$ is a convex combination of the $X_{(i)}$, $i=1, \ldots, m)$. Now, let

$$
\hat{\mu}_{x}=\frac{\sum_{k=1}^{m} X_{(k)}^{(k)}+\sum_{k=1}^{m} X_{\left(i_{k}\right)}^{(k) *}}{2 m} .
$$

Thus, $E\left(\hat{\mu}_{x}\right)=(1 / 2 m)\left(m \mu+\sum_{i=1}^{m} a_{i}^{(k)} \mu_{(i)}\right)=\mu / 2+(1 / 2 m) \sum_{i=1}^{m} a_{i}^{(k)} \mu_{(i)}$; thus $\hat{\mu}_{x}$ can be a biased estimator of $\mu$. Again, let eff be the efficiency of the usual estimator of $\mu$ using RSS of size $m$ w.r.t. the corresponding estimator using SRS; the values of eff are well established for most of the well-known distributions. The efficiency of $\hat{\mu}_{x}$ w.r.t. $\hat{\mu}_{\mathrm{SRS}}$ is given by

$$
\operatorname{eff}\left(\hat{\mu}_{x} ; \hat{\mu}_{\text {SRS }}\right)=\frac{\sigma^{2} / 2 m}{\frac{1}{4}\left[\frac{\sigma^{2}}{m \times \mathrm{eff}}+\operatorname{Var}\left(\frac{1}{m} \sum_{i=1}^{m} a_{i}^{(k)} X_{(i)}\right)\right]+\left(-\frac{\mu}{2}+\frac{1}{2 m} \sum_{k=1}^{m} \sum_{i=1}^{m} a_{i}^{(k)} \mu_{(i)}\right)^{2}} .
$$

eff $\left(\hat{\mu}_{x} ; \hat{\mu}_{\mathrm{SRS}}\right)$ depends on the values of $a_{i}^{(k)}$. If $a_{i}^{(k)}=1 / m$ for all $i$ then $E\left(\hat{\mu}_{x}\right)=\mu$ and $\operatorname{Var}\left(\hat{\mu}_{x}\right)=$ $\left(\sigma^{2} / 4 m\right)(1 /$ eff +1$)$. Thus, in this case

$$
\operatorname{eff}\left(\hat{\mu}_{x} ; \hat{\mu}_{\text {SRS }}\right)=\frac{2 \mathrm{eff}}{1+\mathrm{eff}}
$$

If $a_{i}^{(k)}=0$ for all $i$ except $a_{k}^{(k)}=1$, then the sample is equivalent to two RSSs of size $m$ each; thus, $\operatorname{eff}\left(\hat{\mu}_{x} ; \hat{\mu}_{\mathrm{SRS}}\right)=1$. For other values of $a_{i}^{(k)}$, the efficiency can be obtained similarly.

\section{Application: trees data}

In this section, data on heights $(Y)$ and diameters $(X)$ of 1,083 trees is used to illustrate the last approach of MBVRSS2. These data was due to Pordan (1968). For this data: $\mu_{x}=23.07, \mu_{y}=21.656$, $\sigma_{x}^{2}=6.268, \sigma_{y}^{2}=3.039$, and $\rho=0.721$. To choose different MBVRSS2s from this bivariate data, we followed the steps described previously. For $m=3$ and $m=5,10,000$ MBVRSS2s were chosen randomly; so, 10,000 values were generated for $\hat{\mu}_{x}$ and $\hat{\mu}_{y}$.

- For $m=3$, based on Table 8 we have:

$\operatorname{Bias}\left(\hat{\mu}_{x}\right)=E\left(\hat{\mu}_{x}\right)-\mu_{x} \cong 23.054-23.070=-0.016, \operatorname{MSE}(\bar{X}) \cong \sigma_{x}^{2} / 2 m=6.548, \operatorname{MSE}\left(\hat{\mu}_{x}\right)=$ $(2.032)^{2}+(-0.016)^{2}=4.129$. Thus, eff $\left(\hat{\mu}_{x} ; \bar{X}\right)=\operatorname{MSE}(\bar{X}) / \operatorname{MSE}\left(\hat{\mu}_{x}\right)=1.586$. Similarly, eff $\left(\hat{\mu}_{y} ; \bar{Y}\right)=$ 1.539 .

- For $m=5$, based on Table 9 we have:

$\operatorname{eff}\left(\hat{\mu}_{x} ; \bar{X}\right)=3.929 / 1.991=1.973$ and $\operatorname{eff}\left(\hat{\mu}_{y} ; \bar{Y}\right)=0.924 / 0.462=2$. 
Table 8: The summary of the resulted descriptive statistics for $m=3$

\begin{tabular}{cccccc}
\hline \hline Variable $\downarrow$ & $N$ & Average & Standard deviation & Max & Min \\
\hline$X$ & 1083 & 23.054 & 2.032 & 33.175 & 16.567 \\
$Y$ & 1083 & 21.655 & 0.982 & 25.033 & 17.467 \\
\hline \hline
\end{tabular}

Table 9: The summary of the resulted descriptive statistics for $m=5$

\begin{tabular}{cccccc}
\hline \hline Variable $\downarrow$ & $N$ & Average & Standard deviation & Max & Min \\
\hline$X$ & 1083 & 23.047 & 1.411 & 29.775 & 18.090 \\
$Y$ & 1083 & 21.647 & 0.680 & 24.010 & 18.890 \\
\hline \hline
\end{tabular}

\section{Concluding remarks and suggested future work}

In this paper, two approaches of BVRSS are introduced, which are shown to be more convenient to apply in practice. The first approach was based on ranking of one variable and noting the exact rank of the other variable in one cycle, and do the other way around in the other cycle. The second approach is based on ranking the first variable in the first cycle and give the second variable the same rank (concomitant order statistic), and do the other way around in the second cycle. The two approaches were investigated in general and for some well-known distributions. A real data set was used for illustration. The suggested approaches are shown to be useful for use in practice and can be more efficient than using SRS. The proposed two approaches of BVRSS can be applied on other modifications of RSS such as moving extreme ranked set sampling; this will be another choice that can be investigated in the future. Parametric statistics can be done if the underlying distribution is known; consequently, the MLE can be obtained. The information in the chosen sample can be measured using Fisher information number.

\section{Acknowledgements}

Our sincere thanks is given to the referees for their careful reading of the paper and comments that significantly improved the original version of the paper.

\section{References}

Al-Odat MT and Al-Saleh MF (2001). A variation of ranked set sampling, Journal of Applied Statistical Science, 10, 137-146.

Al-Omari AI and Al-Saleh MF (2009). Quartile double ranked set sampling for estimating the population mean, Stochastics and Quality Control, 24, 243-253.

Al-Saleh MF and Ababneh A (2015). Test for accuracy in ranking in moving extreme ranked set sampling, International Journal of Computational and Theoretical Statistics, 2, 67-77.

Al-Saleh MF and Al-Ananbeh AM (2007). Estimation of the means of the bivariate normal using moving extreme ranked set sampling with concomitant variable, Statistical Papers, 48, 179-195.

Al-Saleh MF and Aldarabseh MZ (2017). Inference on the skew normal distribution using ranked set sampling, International Journal of Computational and Theoretical Statistics, 4, 65-76.

Al-Saleh MF and Al-Hadrami SA (2003a). Parametric estimation for the location parameter for symmetric distributions using moving extremes ranked set sampling with application to trees data, Environmetrics, 14, 651-664.

Al-Saleh MF and Al-Hadrami SA (2003b). Estimation of the mean of the exponential distribution using moving extremes ranked set sampling, Statistical Papers, 44, 367-382. 
Al-Saleh MF and Al-Kadiri MA (2000). Double-ranked set sampling, Statistics \& Probability Letters, 48, 205-212.

Al-Saleh MF and Al-Omari AI (2002). Multistage ranked set sampling, Journal of Statistical Planning and Inference, 102, 273-286.

Al-Saleh MF and Diab YA (2009). Estimation of the parameters of Downton's bivariate exponential distribution using ranked set sampling scheme, Journal of Statistical Planning and Inference, 139, 277-286.

Al-Saleh MF and Na' amneh AK (2014). Properties of the elements of simple, ranked set and moving extreme ranked set samples, Journal of Applied Statistical Science, 22, 75-85.

Al-Saleh MF and Zheng G (2002a). Estimation of bivariate characteristics using ranked set sampling, Australian \& New Zealand Journal of Statistics, 44, 221-232.

Al-Saleh MF and Zheng G (2002b). Modified maximum likelihood estimators based on ranked set sampling, Annals of the Institute of Statistical Mathematics, 54, 641-658.

Downton F (1970). Bivariate exponential distribution in reliability theory, Journal of the Royal Statistical Society. Series B (Methodological), 32, 408-417.

Fery JC (2007). New imperfect ranking models for ranked set sampling, Journal of Statistical Planning and Inference, 137, 1433-1445.

Hanandeh AA and Al-Saleh MF (2013). Inference on Downton's bivariate exponential distribution based on moving extreme ranked set sampling, Austrian Journal of Statistics, 42, 161-179.

McIntyre GA (1952). A method of unbiased selective sampling, using ranked set, Australian Journal of Agriculture Research, 3, 385-390.

Norris RC, Patil GP, and Sinha AK (1995). Estimation of multiple characteristics by ranked set sampling methods, Coenoses, 10, 95-111.

Patil GP, Sinha AK, and Taillie C (1994). Ranked set sampling for multiple characteristics, International Journal of Ecology and Environmental Sciences, 20, 357-373.

Pordan M (1968). Forest Biometric, Pergamum Press, London.

Ridout MS (2003). On ranked set sampling for multiple characteristics, Environmental and Ecological Statistics, 10, 255-262.

Samawi HM and Al-Saleh MF (2007) On bivariate ranked set sampling for ratio and regression estimators, International Journal of Modeling and Simulation, 27, 299-305.

Scheaffer RL, Mendenhall W, Ott RL, and Gerow KG (2011). Elementary Survey Sampling (7th ed), Duxbury Press, London.

Stokes SL (1977). Ranked set sampling with concomitant variables, Communications in StatisticsTheory and Methods, 6, 1207-12011.

Takahasi K and Wakimoto K (1968). On unbiased estimates of the population mean based on the sample stratified by means of ordering, Annals of the Institute of Statistical Mathematics, 20, $1-31$.

Yang SS (1977). General distribution theory of the concomitant of order statistics, The Annals of Statistics, 5, 996-1002.

Zamanzade E and Mohammadi M (2016). Some modified mean estimators in ranked set sampling using a covariate, Journal of Statistical Theory and Applications, 15, 142-152. 